\title{
Application of the Theory of Planned Behavior to Predict Dental Attendance and Caries Experience among Children of Newcomers
}

\author{
Maryam Amin ${ }^{1, *(1)}$, Maryam Elyasi ${ }^{1}$, Babak Bohlouli $^{2}$ and Mohamed ElSalhy ${ }^{3}$ \\ 1 School of Dentistry, Faculty of Medicine and Dentistry, University of Alberta, Edmonton, AB T6G 2R7, \\ Canada; elyasi@ualberta.ca \\ 2 Department of Emergency Medicine, Faculty of Medicine and Dentistry, University of Alberta, Edmonton, \\ AB T6G 2R7, Canada; bohlouli@ualberta.ca \\ 3 College of Dental Medicine, University of New England, Portland, ME 04103, USA; melsalhy@une.edu \\ * Correspondence: maryam.amin@ualberta.ca; Tel.: +1-780-492-7354; Fax: +1-780-492-7536
}

Received: 30 August 2019; Accepted: 25 September 2019; Published: 29 September 2019

\begin{abstract}
Background: This study aimed to explore the predictors of regular dental attendance behavior and caries experience among children of newcomers to Canada using the Theory of Planned Behavior (TPB). Methods: A total of 274 newcomer parents with a child aged 1 to 12 years old who had lived in Canada for 10 years or less participated in this cross-sectional study. Children underwent a dental examination to measure their deft/DEFT, and parents completed a self-administered questionnaire. Parental attitudes, subjective norms, perceived behavioral control (PBC), and intention were examined using Structural Equation Modeling (SEM) as predictors of dental attendance behavior and caries experience using the TPB model. Results: Out of all the components of the model, attitude and PBC significantly predicted the intention $(p<0.05)$ while the subjective norm had no statistical effects on the loading of the model $(p>0.05)$. Intention significantly predicted behavior, but behavior could not predict caries experience. Although $51.6 \%$ of the variance of intention was predicted by this model, only $0.2 \%$ of the variance for dental attendance behavior was explained. Conclusions: The theory of planned behavior was successful in predicting dental attendance intention and behavior for children among newcomers, but this behavior could not predict caries experience in children.
\end{abstract}

Keywords: dental attendance; children; structural equation modeling; theory of planned behavior

\section{Introduction}

Newcomers form a sub-segment of the community that requires special attention due to their unique challenges, like financial insecurity, unemployment, language barriers, and cultural diversity [1,2]. Such differences can also predispose them to suboptimal oral health and oral health-related habits, including care-seeking behavior [3]. Regular dental attendance is a preventive measure to optimize children's oral health [4]. Such habits must be established early after migration to prevent oral health deterioration until families are more settled. However, lower rates of dental visits have been repeatedly reported among newcomers $[1,5]$. Patient-level factors affecting children's dental attendance include parents' education, socioeconomic status, behavioral beliefs, perceived power, and subjective norms [6].

Health behaviors can be predicted and explained by psychosocial theories. The theory of planned behavior (TPB) is one of the applied theories used to predict determinants of health behaviors and develop the interventions used in health promotion programs and behavioral changes [7]. TPB is a socio-cognitive model of the attitude-behavior relationship mediated by behavioral intentions. According to TPB, intention directly influences behavior and is shaped by attitudes, subjective norms, 
and perceived behavioral control of behavior [8]. TBP has been successful in explaining dental attendance behavior via attitude and intention in the general population $[9,10]$. However, little is known about the usefulness of this theory in high-risk groups, such as newcomers to the country, where the relationship between attitude and intention or intention and behaviors may not be as straightforward as in the proposed theory.

The cultural beliefs and perceptions that immigrants bring with them, as well as the level of their acculturation and the way they integrate into their new surroundings, may also influence their care-seeking behavior [11]. Newcomers are transitioning from their original culture into a new culture whose social norms may contradict their traditional cultural norms, and their perceived behavioral control may be influenced by challenges like financial insecurity, unemployment, and language barriers [11,12]. This study aimed to explore the predictors of regular dental attendance behavior and caries experience among children of newcomers using the Theory of Planned Behavior as a theoretical framework.

\section{Methods}

\subsection{Setting and Sampling}

A convenience sample group of immigrant parents with a child age 1-12 who had lived in Canada for 10 years or less was invited to participate in this cross-sectional study. A group of newcomer families and their children were recruited through programs run by the community settlement agencies serving newcomers in Edmonton. All participants signed an informed consent form before participation. The study protocol was approved by the University of Alberta Research Ethics Board and Alberta Health Services (Protocol \# Pro00058397).

\subsection{Data Collection}

Data collection was done in different community locations through events organized by community settlement agencies. Trained bilingual community workers obtained parents' consent and helped with the administration of the questionnaire, which was in English. Almost all parents who were approached accepted the invitation. The questionnaire, developed based on previous studies $[13,14]$, was first tested in a small focus group of 20 mothers with young children to determine the comprehensiveness, understandability, and neutrality of the questions, and required modifications were made. The questionnaire was composed of 2 major sections. The first section included participants' demographics and children's oral hygiene and dietary habits. Collected demographics included the child's and mother's age, the number of children in the family, the child's live-in parent status, the child's place of birth, number of years in Canada, the mother's level of education, household monthly income, and having dental insurance. Questions on the children's oral hygiene and diet included the frequency of brushing (less than twice a day/twice a day or more), the last dental visit (within the past 12 months/over one year/never), the reason for their last dental visit (regular check-up/urgent dental problems/others), and sugar consumption frequency (never/less often than everyday/once a day/twice a day/three times a day or more).

The second section was a validated questionnaire consisting of 24 items based on the Theory of Planned Behaviour (TPB) constructs adopted to examine parental attitudes (8 items), subjective norms (10 items), perceived behavioral control (PBC) (5 items), and intention (1 item) towards their child's dental attendance. Attitude questions included statements about dental visits being traumatic, important, reassuring, unpleasant, and/or if a dental visit decreases caries experience and/or reduces fear toward the dentist. Subjective norms were the evaluated norms based on visiting the dentist early among partners, parents, other family members, friends, and/or family physicians. The perceived control section examined having time, positively preparing the child, and the ability to manage a dental visit twice a year. The intention was measured by the statement "I think of making an appointment with the dentist on time." Responses for the questions were measured on a 7-point Likert scale ranging 
from 1 (strongly disagree) to 7 (strongly agree). The sum of the responses to the items measuring the TPB constructs indicates their final scores. Therefore, a higher total score for items measuring a construct indicated a more positive/stronger construct.

Completion of the questionnaire took about 20 to $30 \mathrm{~min}$. Questionnaires were assessed for completeness on-site, and any missing or incomplete information was immediately collected from the participants.

\subsection{Dental Examination}

A portable dental chair, LED lamp, sterilized mirror, and explorer were used for dental examination of children in the community. The caries status of each child was recorded according to WHO criteria [15] by a calibrated dentist. Caries experience was measured as the total number of decayed teeth, teeth extracted due to caries, and filled teeth (deft/DEFT).

\subsection{Statistical Analysis}

Data analyses were performed using the SPSS 24.0 software (IBM Corp., Armonk, NY, USA). Means, standard deviations, and Pearson's correlations were computed between the study variables. Discrete variables were reported in percentages, and continuous variables were presented as the mean $\pm \mathrm{SD}$, the median, and the range, and compared with the $t$-test (where appropriate). Cronbach's alpha values were calculated to report an estimate of the internal consistency among items of TPB constructs, including attitude, subjective norm (SN), and perceived behavior control (PBC). A confirmatory factor analysis was performed to examine the prior model's (TPB) goodness of fit with the study cohort. A $p$-value of less than 0.05 was considered significant in the analyses.

SPSS AMOS 7.0 (IBM Corp., Armonk, NY, USA) was used for Structural Equation Modeling (SEM) to examine the predictive ability of TPB constructs to predict dental attendance behavior and, subsequently, caries experience. The maximum likelihood was used to estimate the parameters of the model [16]. The adequacy of the model fit was examined using the chi-square test statistic, the comparative fit index (CFI), and the root mean squared error of approximation (RMSEA) [16,17].

\section{Results}

\subsection{Participates Characteristics}

In this study, 274 parents completed the questionnaires with no missing data in the final dataset. $25.6 \%$ of the families were from South Asia (Nepal, India, Burma, Moldova), 23\% were from East Asia (China, Hong Kong, Philippines), 38.2\% were from Africa (Eritrea, Ethiopia, Somalia), and $11.8 \%$ were from East Europe (Ukraine, Russia, Romania). The mean (SD) age of the children was 4.5 years $(\mathrm{SD}=2.9)$, and $55 \%$ were girls. There was no significant age difference according to gender $(p>0.05)$. About $55 \%$ of children were born in Canada, and $85.7 \%$ of them were living with both parents. The mean age of the mothers was 34.9 years $(\mathrm{SD}=6.3)$, and $57.2 \%$ had college or higher education. Of 274 families, $41 \%$ had lived in Canada for less than 5 years and $37.5 \%$ reported a monthly household income of less than $\$ 2000$. The majority of the participants were from China, India, Nepal, Ethiopia, and Eritrea. Of the 274 children, $42.3 \%$ had dental insurance, 34.3\% visited the dentist for check-ups within a year, $62.8 \%$ brushed their children's teeth less than once a day, and $34.7 \%$ consumed sugar twice a day or more. The mean deft/DEFT was 3.3, ranging from 1 to 16 . The participants' characteristics are summarized in Table 1. 
Table 1. Participants characteristics (number of participants: 274).

\begin{tabular}{|c|c|c|c|}
\hline Child's age & & deft/DEFT (Caries experience) & \\
\hline Mean & 4.53 & Mean & 3.28 \\
\hline Median & 4 & Median & 2 \\
\hline SD (range) & $2.98(1-12)$ & SD (range) & $3.76(0-16)$ \\
\hline Children of a female gender $(\mathrm{N})(\%)$ & $150(55.0)$ & $\begin{array}{l}\text { Children with dental insurance } \\
(\mathrm{N})(\%)\end{array}$ & $116(42.3)$ \\
\hline Children born in Canada (N) (\%) & $148(54.9)$ & $\begin{array}{c}\text { Children's last dental visit within } \\
\text { a year }(\mathrm{N})(\%)\end{array}$ & $94(34.3)$ \\
\hline Mother's age & & Reason for visit (N) (\%) & \\
\hline Mean & 34.92 & Check up & $63(23.0)$ \\
\hline Median & 34 & Treatment & $32(11.6)$ \\
\hline \multirow[t]{3}{*}{$\mathrm{SD}$} & \multirow[t]{3}{*}{6.35} & \multicolumn{2}{|l|}{ Tooth brushing frequency (N) (\%) } \\
\hline & & $<$ twice/day & $172(62.8)$ \\
\hline & & $\geq$ twice/day & $102(37.2)$ \\
\hline $\begin{array}{l}\text { Number of Children in family }(\mathrm{N}) \\
(\%)\end{array}$ & \multicolumn{3}{|c|}{ Sugar intake frequency $(\mathrm{N})(\%)$} \\
\hline$\leq 2$ & $218(79.5)$ & $\leq$ once/day & $179(65.3)$ \\
\hline 3 or more & $56(20.5)$ & $\geq$ twice/day & $95(34.7)$ \\
\hline Mother's education level (N) (\%) & \multicolumn{3}{|c|}{ Monthly income (N) (\%) } \\
\hline High school & $117(42.7)$ & $\leq 1999$ & $103(37.5)$ \\
\hline College & $51(18.6)$ & 2000-3999 & $107(39.0)$ \\
\hline University & $106(38.6)$ & $\geq 4000$ & $64(23.3)$ \\
\hline Living with both parents & \multicolumn{3}{|c|}{ Years in Canada $(\mathrm{N})(\%)$} \\
\hline $\mathrm{N}(\%)$ & $235(85.7)$ & $\leq 5$ years & $112(40.9)$ \\
\hline
\end{tabular}

\subsection{Scale Reliability}

A subset of items designed to measure TBP constructs was assessed as follows: attitude through eight 7-point Likert-type items (Cronbach's: 057); subjective norm through nine 7-point Likert-type items (Cronbach's: 0.85); and perceived behavioral control through five 7-point Likert-type items (Cronbach's: 0.62). Given the preliminary reliability values, some items were removed to improve the Cronbach alpha value; therefore, by removing 2 items from the PBC and 2 items from the attitude constructs, the Cronbach alpha increased to 0.738 and 0.742 respectively. All items measuring a subjective norm construct were kept in the analyses. Internal consistency coefficients of more than 0.70 are generally considered acceptable [18]. Taking into account the sensitivity of Cronbach's alpha to a low number of items, the average Cronbach's alpha was over 0.88 and suggested that the scales are homogenous (Appendix A).

\subsection{Structural Equation Modeling}

In the first step, confirmatory factor analyses showed a significant covariance between the latent variables suggesting that the TPB model was appropriate for the study population to predict children's dental attendance behavior and caries experience (Table 2).

Table 2. Covariance between model components.

\begin{tabular}{ccccccc}
\hline & & & Estimate & S.E. & C.R. & P \\
\hline PBC & $<->$ & Subjective Norm & 1.788 & 0.246 & 7.274 & 0.001 \\
PBC & $<->$ & Attitude & 1.366 & 0.188 & 7.247 & 0.001 \\
Subjective Norm & $<->$ & Attitude & 1.405 & 0.174 & 5.630 & 0.001 \\
\hline
\end{tabular}

The SEM showed an acceptable fit based on the measured indices $\left(\mathrm{c}^{2}=376.970, \mathrm{df}=177, p<0.001\right.$, $\mathrm{CMIN} / \mathrm{DF}=2.13, \mathrm{RMSEA}=0.06, \mathrm{CFI}=0.9)$. The SEM results showed that, out of all components of the 
model, the PBC and attitude significantly predicted the intention $(p<0.05)$, and the subjective norm had no statistical effects on the loading of the model. Intention also significantly predicted behavior $(p<0.05)$, but behavior could not predict deft/DEFT. Additionally, there was a significant association between the PBC and behavior in the model (Table 3). A diagram of measurements and structural components of the model is illustrated in Figure 1. Standardized beta coefficients for the measurement and structural parts of the model components are presented in Table 3. The actual diagram of the SEM analyses is presented in Appendix B. In this model, while $51.6 \%$ of the variance accounted for intention, only $0.2 \%$ and $0.1 \%$ of the variances explained dental attendance behavior and deft/DEFT, respectively.

Table 3. Standardized regression weight for the structural parts of the Theory of Planned Behavior (TPB) model components.

\begin{tabular}{|c|c|c|c|c|c|c|}
\hline Items & & & Estimate & SE & CR & $p$-Value \\
\hline PBC_1 & $<\longrightarrow>$ & PBC & 0.579 & 0.099 & 8.861 & 0.001 \\
\hline PBC_2 & $<->$ & PBC & 0.757 & & & \\
\hline PBC_3 & $<\longrightarrow>$ & PBC & 0.774 & 0.076 & 11.698 & 0.001 \\
\hline Subjective norm_1 & $<\longrightarrow>$ & Subjective norm & 0.604 & 0.094 & 8.676 & 0.001 \\
\hline Subjective norm_2 & $<->$ & Subjective norm & 0.731 & 0.099 & 10.179 & 0.001 \\
\hline Subjective norm_3 & $<\longrightarrow>$ & Subjective norm & 0.658 & & & \\
\hline Subjective norm_4 & $<->$ & Subjective norm & 0.635 & 0.071 & 12.074 & 0.001 \\
\hline Subjective norm_5 & $<\longrightarrow>$ & Subjective norm & 0.704 & 0.103 & 9.877 & 0.001 \\
\hline Subjective norm_6 & $<->$ & Subjective norm & 0.166 & 0.087 & 3.107 & 0.002 \\
\hline Subjective norm_7 & $<->$ & Subjective norm & 0.472 & 0.098 & 6.965 & 0.001 \\
\hline Subjective norm_8 & $<->$ & Subjective norm & 0.399 & 0.094 & 6.371 & 0.001 \\
\hline Subjective norm_9 & $<->$ & Subjective norm & 0.436 & 0.11 & 6.466 & 0.001 \\
\hline Attitude_1 & $<->$ & Attitude & 0.609 & 0.091 & 10.127 & 0.001 \\
\hline Attitude_2 & $<->$ & Attitude & 0.601 & 0.089 & 9.969 & 0.001 \\
\hline Attitude_3 & $<\longrightarrow$ & Attitude & 0.842 & & & \\
\hline Attitude_4 & $<->$ & Attitude & 0.29 & 0.101 & 4.482 & 0.001 \\
\hline Attitude_5 & $<->$ & Attitude & 0.593 & 0.077 & 10.407 & 0.001 \\
\hline Last child dental visit & $<\longrightarrow>$ & Behavior & -0.228 & 0.292 & -0.208 & 0.835 \\
\hline Reason for dental visit & $<->$ & Behavior & 3.321 & & & \\
\hline Intention & $<\longrightarrow$ & Subjective norm & -0.301 & 0.241 & -1.598 & 0.11 \\
\hline Intention & $<\longrightarrow>$ & Attitude & 0.61 & 0.167 & 5.032 & 0.001 \\
\hline Intention & $<\longrightarrow>$ & PBC & 0.441 & 0.174 & 2.904 & 0.004 \\
\hline Behavior & $<->$ & PBC & 0.042 & 0.022 & 1.993 & 0.046 \\
\hline Behavior & $<->$ & Intention & 0.049 & 0.019 & -2.365 & 0.018 \\
\hline DEFT/deft & $<\longrightarrow$ & Behavior & -0.037 & 0.439 & -0.197 & 0.844 \\
\hline
\end{tabular}

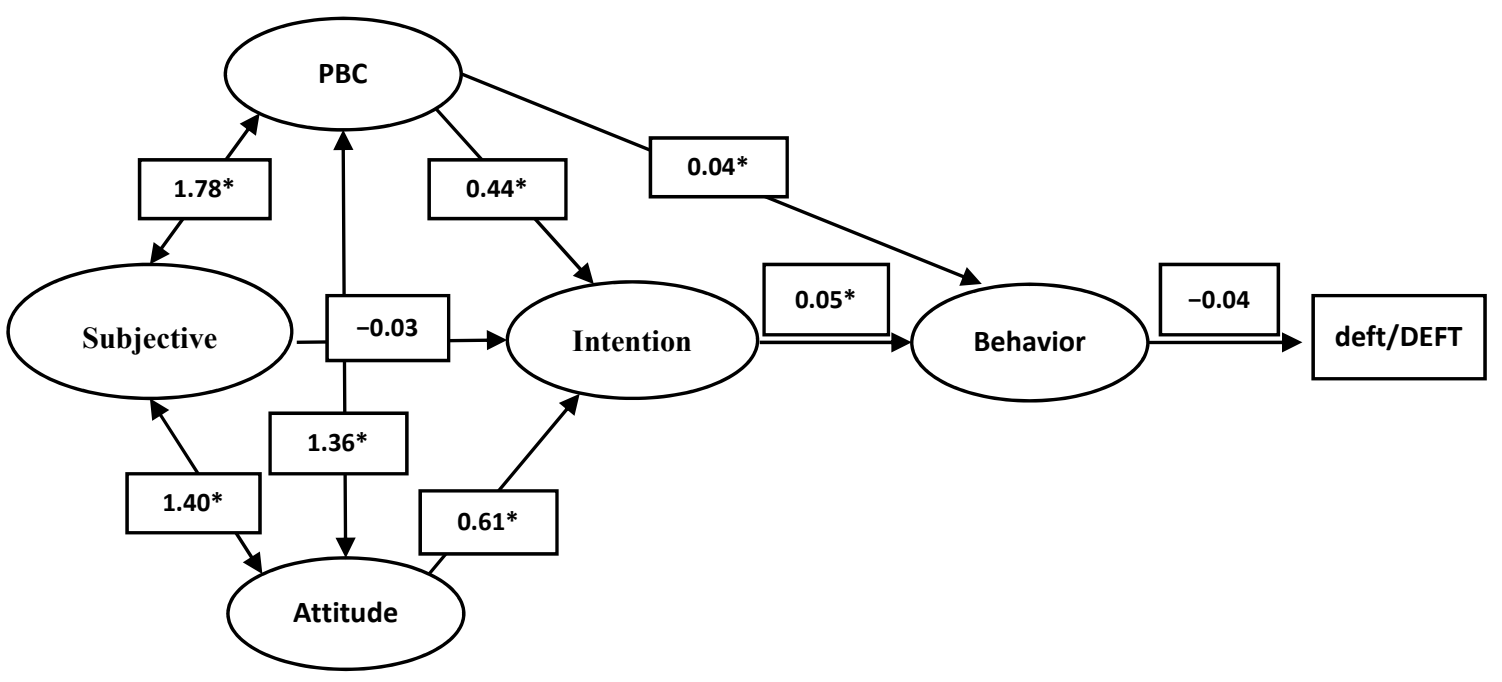

Figure 1. Causal model path beta coefficients, ${ }^{*} p<0.05$. 


\section{Discussion}

This study applied TPB as a model to predict regular dental attendance behavior and caries experience among newcomers' children using a Structural Equation Modeling approach. The attitude and PBC components of TPB significantly predicted parental intention to have regular dental visits for their children. Subjective norms had no statistical effects on the loading of the model. Intention also significantly predicted dental attendance behavior, but the behavior could not predict caries experience. Only $0.2 \%$ of the variance in dental attendance behavior was explained by intention.

The proportion of dental attendance within the previous year was about $34 \%$ among children of newcomers. This prevalence is lower than the previously reported $51 \%$ among 378 preschoolers in Edmonton, Alberta [13]. A higher proportion of participants in the present study reported a monthly income of less than 1999 CAD compared to the general population (37.5\% vs. $21.6 \%)$. In addition, participants in the present study had lower rates of dental insurance compared to the general population ( $42.3 \%$ vs. $74.8 \%$ ). This could be an explanation for the differences in dental attendance rates. Dental costs and insurance were the most commonly reported reasons behind suboptimal use of dental services, as they can restrict access to dental care [6].

Attitudes toward different behaviors have always been considered one of the strongest determinants of these behaviors [7]. In the present study, both attitude and PBC accurately predicted intention. This finding supports other studies that predict decisions to utilize dental care $[9,10]$. This highlights the importance of oral health education and promotion programs targeting both attitude and PBC toward dental attendance [19]. Interestingly, the subjective norm construct did not predict intention. A potential explanation is that newcomers are transitioning from their original cultures into a new culture, where subjective norms may contradict new cultural norms [11].

Intention was translated into a behavior among participants of the present study. Although the pathway was statistically significant, the variance of behavior explained in the model was very low $(0.2 \%)$. This could be attributed to the minimal number of items adopted in the study to measure participants' intentions in the present study. In addition, the presence of socioeconomic and/or structural barriers may affect the translation of intention to behavior [7]. Financial insecurity, unemployment, language barrier, transportation, and navigating the healthcare system have been reported as factors affecting care-seeking behavior among newcomers [6]. There is no conclusive literature on which specific barriers will mostly affect the translation of intention to behavior [7]. However, this difference highlights the importance of affordable, culturally appropriate programs that focus on vulnerable populations to prevent deterioration of their oral health and keep them aligned with the majority population.

Regular dental attendance provides an opportunity to assess patients' caries risk, provide oral health education, and provide professional preventive care and treatments. Having a dental visit is a proxy measure of access to dental care. Although a routine biannual dental visit is the most recommended practice, this practice has been challenged because of the lack of evidence [4]. Multiple Cochrane reviews concluded that there is the lack of evidence to support or refute the benefits of dental check-ups every 6 months or to demonstrate any harmful effects of longer recall intervals $[19,20]$. In the present study, regular dental attendance could not predict caries experience measured as deft/ DEFT. The complexity of caries etiology may be the main explanation for this result [21]. Diet, oral hygiene behaviors and patient susceptibility are important contributors to caries formation [21]. Although there is a lack of evidence regarding the benefits of specific recall intervals, accessing dental care might have a generally positive impact on quality of life [22]. It was shown that regular users of dental services have better OHRQoL compared to non-regular users [22].

While the TPB showed success in explaining dental attendance behavior through attitude and intention in the general population $[9,10]$, its prediction was not strong among the immigrant population in this study (i.e., only $0.2 \%$ of the variance of dental attendance behavior was explained). Using a different health behavior model/theory and/or expansion of the TPB can be valuable to understand the determinants/predictors of dental attendance among newcomers. Some other behavioral constructs 
have been proposed to predict oral health behaviors and can be added to the model. The Sense of Coherence, for example, was a significant determinant of oral health-related behaviors, including tooth brushing frequency, daily smoking, and dental attendance $[13,23]$. Sense of Coherence (SOC) describes the capability of individuals to use existing resources in order to overcome difficulties and cope with life stressors to perform healthy behaviors [24]. Immigrant individuals with a stronger SOC may be able to transfer their intentions to behaviors. If this is true, newcomers may benefit from programs that improve SOC through skill training. However, this must be examined in future studies.

One limitation of the present study is its convenient sample group. Although all clients who attended the settlement agency activities were invited, the ones who participated might have been the most affected by dental care barriers. However, a random sampling of the immigrant population is not achievable as this population is scattered among the general population and across the province. Another limitation was the use of self-administered English language questionnaire that could affect participants' understanding of the questions. To avoid misinterpretation of the questions, bilingual translators were available on-site to help the participants if needed.

SEM was used in the present study to examine how TPB constructs predict dental attendance behavior and caries experience. SEM has advantages over a basic multivariate regression analysis in its ability to simultaneously test the hypothesized relationships among observed and latent variables in the model. However, similar to other statistical methods, SEM has its limitations. Although a latent variable can be a closer approximation of a construct than a measured variable in the model, it may not be a clear representation of the construct [25]. Although we used the term effect while explaining the SEM results, this does not mean that our proposed Structural Equation Model is a causal model due to the cross-sectional design of this study. Future longitudinal studies will be needed to explore the causality effects of the TPB construct on the exhibition of the actual behavior instead of self-reported past behavior.

In conclusion, the theory of the planned behavior was successful in predicting regular dental attendance behavior among the children of newcomers, but their behavior could not predict caries experience. Parental attitude and PBC were the main predictors for intention to engage in regular dental attendance. Future studies should work on either expanding the TPB or use of theories/models that include interpersonal, community, or environmental constructs to better predict dental attendance behavior and caries experience among newcomers.

Author Contributions: Conceptualization, M.A.; Data curation, M.E. (Maryam Elyasi) and M.E. (Mohamed ElSalhy); Formal analysis, B.B.; Methodology, M.A. and M.E. (Mohamed ElSalhy); Project administration, M.E. (Maryam Elyasi); Supervision, M.A.; Writing—original draft, M.E. (Mohamed ElSalhy); Writing-review and editing, M.A., M.E. (Maryam Elyasi), and B.B.

Funding: This research was funded by School of Dentistry Oral Health Community Engagement Fund ZN004.

Acknowledgments: Authors are thankful to Multicultural Health Brokers COOP for helping with the recruitment of our participants and Ida Kornerup for helping with dental examinations.

Conflicts of Interest: All authors declare no conflict of interest. 
Appendix A Scale Reliability Measure

\begin{tabular}{cccccc}
\hline Item & $\begin{array}{c}\text { Scale Mean If } \\
\text { Item Deleted }\end{array}$ & $\begin{array}{c}\text { Scale Variance } \\
\text { If Item } \\
\text { Deleted }\end{array}$ & $\begin{array}{c}\text { Corrected } \\
\text { Item-Total } \\
\text { Correlation }\end{array}$ & $\begin{array}{c}\text { Squared } \\
\text { Multiple } \\
\text { Correlation }\end{array}$ & $\begin{array}{c}\text { Cronbach's } \\
\text { Alpha If Item } \\
\text { Deleted }\end{array}$ \\
\hline PBC_1 & 85.01 & 360.271 & 0.436 & 0.380 & 0.884 \\
PBC_2 & 84.31 & 356.023 & 0.576 & 0.498 & 0.878 \\
PBC_3 & 83.91 & 357.743 & 0.653 & 0.585 & 0.876 \\
Subjective norm_1 & 83.99 & 362.047 & 0.548 & 0.446 & 0.879 \\
Subjective norm_2 & 84.08 & 353.942 & 0.656 & 0.513 & 0.876 \\
Subjective norm_3 & 84.70 & 349.850 & 0.638 & 0.577 & 0.876 \\
Subjective norm_4 & 84.85 & 357.151 & 0.620 & 0.547 & 0.877 \\
Subjective norm_5 & 84.25 & 351.559 & 0.653 & 0.464 & 0.875 \\
Subjective norm_6 & 85.15 & 358.736 & 0.478 & 0.593 & 0.882 \\
Subjective norm_7 & 84.92 & 367.513 & 0.427 & 0.309 & 0.884 \\
Subjective norm_8 & 84.45 & 353.897 & 0.591 & 0.524 & 0.878 \\
Subjective norm_9 & 84.83 & 359.649 & 0.466 & 0.540 & 0.883 \\
Attitude_1 & 83.91 & 363.959 & 0.494 & 0.374 & 0.881 \\
Attitude_2 & 84.32 & 367.492 & 0.460 & 0.392 & 0.883 \\
Attitude_3 & 83.49 & 364.573 & 0.644 & 0.554 & 0.877 \\
Attitude_4 & 84.78 & 384.936 & 0.195 & 0.242 & 0.892 \\
\hline Attitude_5 & 83.91 & 364.703 & 0.554 & 0.486 & 0.879 \\
\hline
\end{tabular}

\section{Appendix B AMOS Model Diagram}

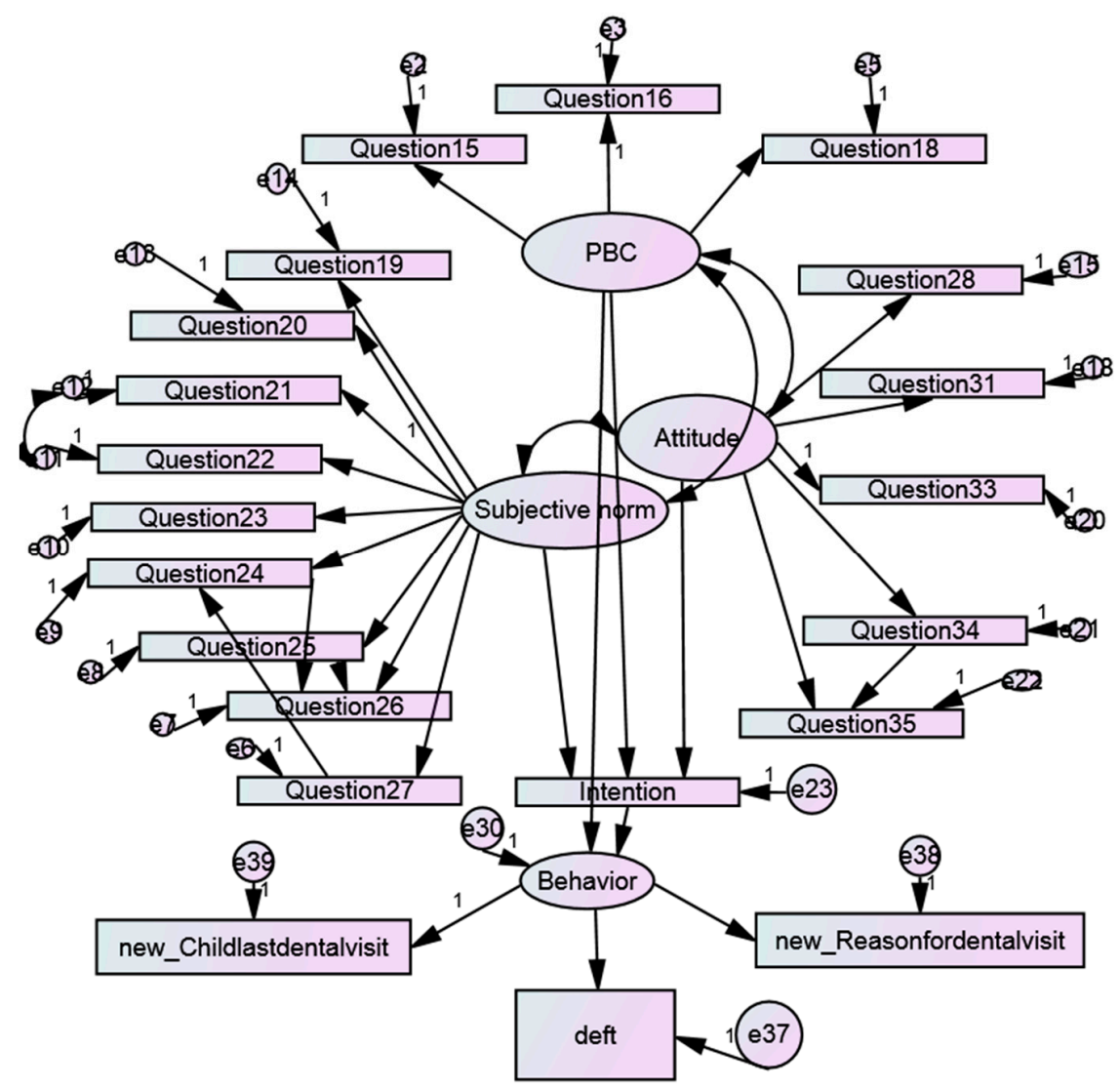

\section{References}

1. Amin, M.; Perez, A.; Nyachhyon, P. Parental Awareness and Dental Attendance of Children Among African Immigrants. J. Immigr. Minority Health Cent. Minor. Public Health 2013. [CrossRef] [PubMed] 
2. Vargas, C.M.; Ronzio, C.R. Disparities in early childhood caries. BMC Oral Health 2006, 6. [CrossRef] [PubMed]

3. Gao, X.L.; McGrath, C. A review on the oral health impacts of acculturation. J. Immigr. Minor. Health Cent. Minor. Public Health 2011, 13, 202-213. [CrossRef] [PubMed]

4. Sheiham, A. Is there a scientific basis for six-monthly dental examinations? Lancet 1977, 2, 442-444. [CrossRef]

5. Newbold, K.B.; Patel, A. Use of dental services by immigrant Canadians. J. Can. Dent. Assoc. 2006, 72, 143. [PubMed]

6. Badri, P.; Saltaji, H.; Flores-Mir, C.; Amin, M. Factors affecting children's adherence to regular dental attendance: A systematic review. J. Am. Dent. Assoc. 2014, 145, 817-828. [CrossRef]

7. Armitage, C.J.; Conner, M. Efficacy of the Theory of Planned Behaviour: A meta-analytic review. Br. J. Soc. Psychol. 2001, 40, 471-499. [CrossRef]

8. Ajzen, I. The theory of planned behavior. Organ. Behav. Hum. Decis. Process. 1991, 50, 179-211. [CrossRef]

9. Astrom, A.N.; Lie, S.A.; Gulcan, F. Applying the theory of planned behavior to self-report dental attendance in Norwegian adults through structural equation modelling approach. BMC Oral Health 2018, 18, 95. [CrossRef]

10. Luzzi, L.; Spencer, A.J. Factors influencing the use of public dental services: An application of the Theory of Planned Behaviour. BMC Health Serv. Res. 2008, 8, 93. [CrossRef]

11. Dahlan, R.; Badri, P.; Saltaji, H.; Amin, M. Impact of acculturation on oral health among immigrants and ethnic minorities: A systematic review. PLoS ONE 2019, 14, e0212891. [CrossRef] [PubMed]

12. Amin, M.; ElSalhy, M. Factors Affecting Dental Attendance of Children of New Immigrant Parents: A Cross-Sectional Study. J. Immigr. Minority Health Cent. Minor. Public Health 2017, 19, 1351-1361. [CrossRef] [PubMed]

13. Elyasi, M.; Abreu, L.G.; Olsen, C.; Baker, S.R.; Lai, H.; Major, P.W.; Amin, M. Parent's Sense of Coherence and Children's Oral Health-Related Behaviors: Is There an Association? Pediatr. Dent. 2018, 40, 23-29. [PubMed]

14. Van den Branden, S.; Van den Broucke, S.; Leroy, R.; Declerck, D.; Hoppenbrouwers, K. Measuring determinants of oral health behaviour in parents of preschool children. Community Dent. Health 2013, 30, 19-25. [PubMed]

15. World Health Organization. Oral Health Surveys: Basic Methods, 5th ed.; World Health Organization: Geneva, Switzerland, 2013.

16. Brown, T.A. Confirmatory Factor Analysis for Applied Research, 2nd ed.; The Guilford Press: New York, NY, USA, 2015; p. 462.

17. Hu, L.; Bentler, P.M. Cutoff criteria for fit indexes in covariance structure analysis: Conventional criteria versus new alternatives. Struct. Equ. Model. A Multidiscip. J. 1999, 6, 1-55. [CrossRef]

18. Santos, J.R.A. Cronbach's alpha: A tool for assessing the reliability of scales. J. Ext. 1999, 37, 88-92.

19. Beirne, P.; Clarkson, J.E.; Worthington, H.V. Recall intervals for oral health in primary care patients. Cochrane Database Syst. Rev. 2007. [CrossRef]

20. Riley, P.; Worthington, H.V.; Clarkson, J.E.; Beirne, P.V. Recall intervals for oral health in primary care patients. Cochrane Database Syst Rev. 2013. [CrossRef]

21. Fisher-Owens, S.A.; Gansky, S.A.; Platt, L.J.; Weintraub, J.A.; Soobader, M.J.; Bramlett, M.D.; Newacheck, P.W. Influences on children's oral health: A conceptual model. Pediatrics 2007, 120, 510-520. [CrossRef]

22. Crocombe, L.A.; Broadbent, J.M.; Thomson, W.M.; Brennan, D.S.; Poulton, R. Impact of dental visiting trajectory patterns on clinical oral health and oral health-related quality of life. J. Public Health Dent. 2012, 72, 36-44. [CrossRef]

23. Elyasi, M.; Abreu, L.G.; Badri, P.; Saltaji, H.; Flores-Mir, C.; Amin, M. Impact of Sense of Coherence on Oral Health Behaviors: A Systematic Review. PLoS ONE 2015, 10, e0133918. [CrossRef] [PubMed]

24. Antonovsky, A. The structure and properties of the sense of coherence scale. Soc. Sci Med. 1993, 36, 725-733. [CrossRef]

25. Beran, T.N.; Violato, C. Structural equation modeling in medical research: A primer. BMC Res. Notes 2010, 3, 267. [CrossRef] [PubMed]

(C) 2019 by the authors. Licensee MDPI, Basel, Switzerland. This article is an open access article distributed under the terms and conditions of the Creative Commons Attribution (CC BY) license (http://creativecommons.org/licenses/by/4.0/). 